\title{
ICHTHYODIVERSITY OF SAN JOSE, SAN FRANCISQUITO, AND EL PARDITO ISLANDS IN THE SOUTHWESTERN GULF OF CALIFORNIA, MEXICO
}

\author{
Deivis S. PALACIOS-SALGADO ${ }^{1 *}$, Xchel G. MORENO-SANCHEZ $^{2}$, \\ Leonardo A. ABITIA-CARDENAS ${ }^{2}$, Francisco J. GUTIERREZ-SANCHEZ ${ }^{2}$, \\ and Jesus RODRIGUEZ-ROMERO ${ }^{3}$
}

${ }^{1}$ Escuela Nacional de Ingeniería Pesquera, Colección ictiológica, Apartado Postal 10, San Blas, Nayarit, México 63740

${ }^{2}$ Centro Interdisciplinario de Ciencias Marinas, Departamento de Pesquerias y Biologia Marina, Apartado Postal 592, La Paz, Baja California Sur, Mexico

${ }^{3}$ Centro de Investigaciones Biologicas del Noroeste, Apartado Postal 128, La Paz, Baja California Sur, Mexico

Palacios-Salgado D.S., Moreno-Sanchez X.G., Abitia-Cardenas L.A., Gutierrez-Sanchez F.J., RodriguezRomero J. 2012. Ichthyodiversity of San Jose, San Francisquito, and El Pardito islands in the southwestern Gulf of California, Mexico. Acta Ichthyol. Piscat. 42 (3): 177-191.

\begin{abstract}
Background. The San Jose and San Francisquito Islands and El Pardito Islet are important for recreational tourism, sport fishing, and commercial fishing. They are part of an "Area Natural Protegida" and "Area Prioritaria Marina"; however, studies on the composition and diversity of their marine floral and faunal communities are limited. To provide information vital for management and conservation of these islands, a check-list of the ichthyofauna was compiled based on original data and the data extracted from other sources.

Materials and methods. Field methodology involved direct collecting of the specimens using a gill net or a charalera net and/or visual censuses. Records of fish present near the islands were either directly solicited from museum curators of national and international scientific collections or obtained by using online database collections. Additionally, an exhaustive literature search for fish records was carried out, including scientific articles, books, and theses. Finally, the preferential habitat and zoogeographic affinity of fishes were analyzed..

Results. The data yielded 298 species representing 201 genera and assigned to 86 families, 36 orders, and two fish classes. The most numerous families in terms of the species number were Serranidae (23 species), Gobiidae (15 species), and Carangidae (14 species). The most numerous genera were Lutjanus and Halichoeres, with eight and six species, respectively. On average, there were 3.5 species per family. The fish community was dominated by tropical eurythermal species with wide distribution in the Eastern Pacific. An important component of endemic Cortez Province species was found, represented by 24 species from the families Chaenopsidae, Gobiidae, Labrisomidae, Tripterygiidae, Gobiesocidae, Dactyloscopidae, Kyphosidae, Opistognathidae, and Ophidiidae.

Conclusion. The fish richness of the San Jose and San Francisquito Islands and El Pardito Islet represents 32\% of the ichthyofauna recorded for the Gulf of California and includes 24 endemic species.
\end{abstract}

Keywords: checklist, Cortez Province, endemic species, Serranidae, Abudefduf troschelii

\section{INTRODUCTION}

The Gulf of California is one of the most diverse regions in the Eastern Tropical Pacific (ETP), having about 911 species, 92 of which are endemic (Thomson et al. 2000, Hastings et al. 2010). This richness has caused the Gulf of California to be considered a place of origin or accumulation of species (Mora and Robertson 2005). Species richness in the Gulf of California is favoured by the topographic and bathymetric complexity that provides a great variety of habitats (Castro-Aguirre et al. 1995, Thomson et al. 2000, Thomson and Gilligan 2002).
The Gulf of California islands have productive environments with high carrying capacities, and are the habitat of a great richness, diversity, and density of fishes (Thomson and Gilligan, 2002). San Jose Island $\left(24^{\circ} 52^{\prime} 12^{\prime \prime}-25^{\circ} 06^{\prime} 02^{\prime \prime} \mathrm{N}, 110^{\circ} 43^{\prime} 03^{\prime \prime}-110^{\circ} 31^{\prime} 58^{\prime \prime} \mathrm{W}\right)$, San Francisquito Island $\left(24^{\circ} 50^{\prime} \mathrm{N}, 110^{\circ} 35^{\prime} \mathrm{W}\right)$ and El Pardito Islet $\left(24^{\circ} 51^{\prime} 10^{\prime \prime} \mathrm{N}, 110^{\circ} 34^{\prime} 52^{\prime \prime} \mathrm{W}\right)$ are located north of $\mathrm{La}$ Paz Bay, and are separated from the peninsula by the narrow San Jose Channel (Holguin-Quiñones et al. 2008). The El Pardito Islet is permanently inhabited by a family of fishermen, who also carry out biodiversity conservation

\footnotetext{
* Correspondence: Dr. Deivis S. Palacios Salgado, Escuela Nacional de Ingeniería Pesquera, Colección ictiológica, Apartado Postal 10, San Blas, Nayarit. México 63740, phone: (+323) 231-21-20, e-mail: palaciossalgado@gmail.com.
} 
activities on the islet. These islands are in a highly productive fisheries zone; their inputs represent more than $60 \%$ of the captures of La Paz Bay (Ramirez-Rodriguez 1997). Commercial catches in this zone undergo important changes at the seasonal, spatial, and fishing gear levels. Specific composition consists of a reduced group of target species and includes several bycatch or occasional species (Montoya-Campos, unpublished*).

These islands also support a great biodiversity of marine macroinvertebrates (Holguin-Quiñones et al. 2008) and mangrove zones (Ramirez-Garcia and Lot 1994) that serve as breeding areas for a wide variety of fish. They are also reproductive areas for several marine bird species (Lozano et al. 2004), and are important for sport fishing as well as tourism and recreational activities such as kayaking. However, despite being part of protected ecological zones, such as the Area Natural Protegida (Anonymous 1978) and Area Prioritaria Marina "Islas del Golfo de California" established by CONABIO (Arriaga et al. 1998), studies on its composition and diversity of marine communities are limited; to date, there are no complete inventories of the fish composition for the area. The majority of the ecosystem ichthyofauna inventories of the Gulf of California have focussed on lagoon systems (e.g., Rodríguez-Romero et al. 1992, Abitia-Cárdenas et al. 1994), and only recently have systematic lists of insular ecosystems been done (Erisman et al. 2011, Del Moral-Flores, unpublished $^{* *}$ ).

In this context, and aiming to provide better information for accurate management and conservation of resources for these islands, this study presents an integrated systematic list of the ichthyofauna from San Jose and San Francisquito Islands and El Pardito Islet. Additionally, the most abundant species are indicated, the preferential habitat and the species' zoogeographic affinity are analyzed.

\section{MATERIALS AND METHODS}

To generate the systematic list of the ichthyofauna from San Jose, San Francisquito, and El Pardito Islands, three information sources were considered: records from field observations, records from both national and international scientific collections, and the scientific literature.

Field methodology consisted of specimen collecting (using a gill net and a charalera net) and of visual censuses. For net sampling, six bimonthly field trips were made from September 2000 to August 2001, and eleven localities were evaluated, eight with gill net and three with charalera net (Fig. 1). The gill net was made of nylon (N.47) monofilament fishing line, and measured $140 \mathrm{~m}$ in length by $3 \mathrm{~m}$ in width, featuring a $9 \mathrm{~cm}$ stretched mesh size. This net was placed obliquely to the shoreline at sunset $(1800 \mathrm{~h})$ and recovered the next day in the morning $(0600 \mathrm{~h})$. The charalera net was $50 \mathrm{~m}$ long and $2 \mathrm{~m}$ wide with a $0.5 \mathrm{~cm}$ stretched mesh size. Net-captured fish were counted, weighed, measured, and identified in the field using the identification key by Fischer et al. (1995). Two organisms of each doubtful species were fixed in $10 \%$ for- malin for species corroboration at the Laboratorio de Ecologia de Peces del Centro Interdisciplinario de Ciencias Marinas (CICIMAR) in La Paz, Baja California Sur, Mexico.

Visual censuses were carried out during ten monthly trips to 13 localities dominated by rocky reefs, from March 2001 to August 2002 (except for January and September) (Fig. 1). These censuses were carried out using SCUBA and free diving, swimming parallel to shore along $50 \mathrm{~m}$ by $5 \mathrm{~m}$ duplicate transects $\left(500 \mathrm{~m}^{2}\right.$ total per station). At the stations of El Melon, El Faro, El Pardito, El Conejo, and San Francisquito 1, the sampling was done at two depths $(1 \sim 3 \mathrm{~m}$ and $5 \sim 7 \mathrm{~m})$. Censuses were made during the day between $1000 \mathrm{~h}$ and $1600 \mathrm{~h}$, which is when outdoor lighting for viewing and identifying fish is the best. All fish species and their abundances were recorded on acrylic sheets. In March, May, September, and October 2011, censuses were carried out in three other localities at two depths using the diving methodology outlined above (Fig. 1).

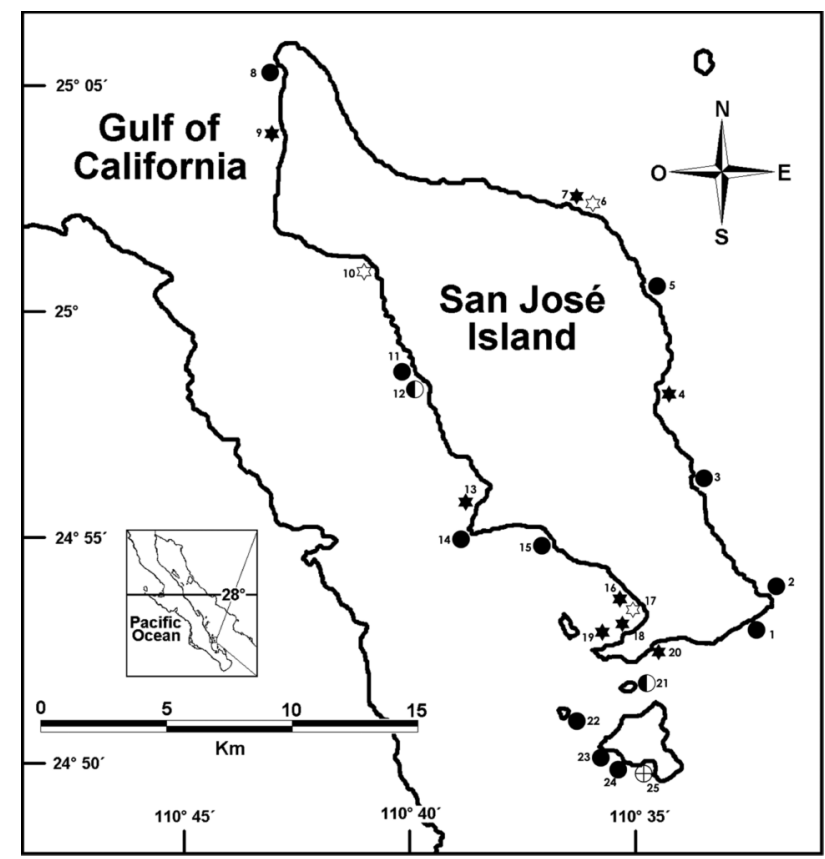

Fig. 1. Evaluated localities from San Jose and San Francisquito Islands, and El Pardito Islet in the southwestern Gulf of California: $1=$ El Conejo, $2=$ La Estacuda, $3=$ El Cordon Negro, $4=$ La Cañada, $5=$ Punta Colorada 1, $6=$ Punta Colorada 2, $7=$ Punta Colorada 3, $8=$ Piedra Ahogada, 9 = Punta Calabozo, $10=$ Punta Cardon, $11=$ La Cuevita, $12=$ El Melon, $13=$ El Muelle, $14=$ El Faro 1, 15 =El Faro 2, $16=$ Boca Estero, $17=$ Boca estero Este, 18 = Boca estero Oeste, $19=$ Punta Cantos, 20 = Boca estero Sur, $21=$ El Pardito, $22=$ El Cayuco, $23=$ San Francisquito 1, $24=$ San Francisquito 2, $25=$ El Cubo del Dandy; Circles represent localities evaluated with visual censuses; black dots - were evaluated during 2001-2002, cross out circles-during 2011, and black-and-white dots-during both periods; black stars represent localities evaluated using a gill net, hollow stars - using a charalera net

\footnotetext{
${ }^{*}$ Montoya-Campos M. 2009. Cambios en la composicion especifica de la captura artesanal de escama al sur de la Isla San Jose, Baja California Sur, Mexico. MSc Thesis. CICIMAR-IPN., La Paz, B.C.S. Mexico.

** Del Moral-Flores L.F. 2010. Diversidad y patrones biogeográficos de la ictiofauna asociada a los complejos insulares del Golfo de California. MSc Thesis. CICIMAR-IPN., La Paz, Mexico.
} 
Records of fish from the islands were solicited directly from the museum curators of national and international scientific collections (e.g., MHNUABCS-CI = Museo de Historia Natural de la Universidad Autonoma de Baja California Sur, La Paz), or obtained by using online databases (e.g., http://www.coleccion.cicimar.ipn.mx); information for twelve of these was found (Table 1). Additionally, an exhaustive search for fish records was carried out in the literature, including scientific papers, books, and theses.

We performed an overview of the fish composition of the islands, and the preferential habitats were recorded. According to their habitat, species were classified as follows: reef (A); soft bottom demersal (B); mixed bottom demersal (C); pelagic-demersal (D; meaning: demersal but occasionally migrating into the water columns); neritic-pelagic (E); oceanic-pelagic (F); mesopelagic (G); bathybenthonic $(\mathrm{H})$; and pelagic-benthonic (I). Biological information was obtained from specific published sources (e.g., Thomson et al. 2000, Robertson and Allen 2008).

Additionally, zoogeographic affinity was analyzed according to the basic scheme by Briggs (1974), with modifications by Hastings (2000), Robertson et al. (2004), and Horn et al. (2006). The species were grouped into the following classes, which take their natural distributions into account:

- Province of San Diego (PS): from Point Conception to Bahía Magdalena off the western coast of Baja California Sur, corresponding to warm-temperate waters, in which the temperature rarely drops below $10^{\circ} \mathrm{C}$ or exceeds $25^{\circ} \mathrm{C}$.

- Cortez Province (PC): including the southern part of Bahía Magdalena (lat $\sim 25^{\circ} \mathrm{N}$ ) and the central and northern Gulf of California). The water temperature in winter rarely falls below $13-15^{\circ} \mathrm{C}$, but in summer it can increase up to $25^{\circ} \mathrm{C}$ or even $30^{\circ} \mathrm{C}$. Along the eastern
Gulf coast, the PC is isolated from the Mexican province by the Sinaloa gap, a $370 \mathrm{~km}$ band of sandy and muddy coastline extending between Topolobampo and Mazatlan.

- Mexican Province (PM): includes the coast of Mexico from Mazatlan, Sinaloa to the Isthmus of Tehuantepec, Oaxaca. This province is located in the tropic, where water temperatures in winter rarely fall below $18-20^{\circ} \mathrm{C}$. It is separated from the PC to the west by a $300 \mathrm{~km}$ stretch of open water between Mazatlan and the Peninsula of Baja California, and to the north by the aforementioned Sinaloa gap.

- Panamic Province (PP): extends south from El Salvador to near Cape Blanco in northern Peru. Between this province and the previous, there is an extensive section (the Central America gap) of sandy coastline (1000 km) between the Gulf of Tehuantepec and El Salvador. Along the southern boundary of this province (lat $\sim 6^{\circ} \mathrm{S}$ ), there is a transition zone influenced by warm waters from Ecuador and cold coastal waters from the Peruvian Current coming from Chile (Chirichigno and Cornejo 2001). This province also has a tropical environment.

- Peruvian-Chilean Province (PPCH): extends from Sechura Bay to northern Chiloe Island in Chile.

- Circumtropical (CT): are the fish species widely distributed in the tropical seas of the world.

- Transpacific (T): species are distributed on both sides of the barrier of the Pacific, Eastern Tropical Pacific (ETP) and Central and Western Tropical Pacific.

- Amfiamerican (AM): species are distributed on both sides of Central America: ETP and Western Atlantic.

Finally, the systematic arrangement followed criteria proposed by Nelson (2006) with modifications by Wiley and Johnson (2010). Genera and respective species are presented alphabetically. The proper spelling of scientific names and common names follow FishBase (Froese and Pauly 2012).

Table 1

Systematic list of the ichthyofauna from San Jose and San Francisquito Islands, and El Pardito Islet in the southwestern Gulf of California

\begin{tabular}{|c|c|c|c|c|}
\hline Species & $\begin{array}{l}\text { Field } \\
\text { records }\end{array}$ & Record code & Habitat & $\begin{array}{l}\text { Ichthyogeographic } \\
\text { affinities }\end{array}$ \\
\hline \multicolumn{5}{|l|}{ CLASS CHONDRICHTHYES } \\
\hline \multicolumn{5}{|l|}{ FAMILY HETERODONTIDAE } \\
\hline Heterodontus francisci (Girard, 1855) & & SIO & $\mathrm{C}$ & $\mathrm{SP}-\mathrm{CP}$ \\
\hline $\begin{array}{l}\text { Heterodontus mexicanus Taylor } \\
\text { et Castro-Aguirre, } 1972\end{array}$ & & LACM & $\mathrm{C}$ & CP-PP \\
\hline \multicolumn{5}{|l|}{ FAMILY TRIAKIDAE } \\
\hline Mustelus henlei (Gill, 1863) & & 4 & $\mathrm{D}$ & OP-CP, PP \\
\hline Mustelus lunulatus Jordan et Gilbert, 1882 & $\mathrm{~b}$ & & $\mathrm{D}$ & OP-PP \\
\hline \multicolumn{5}{|l|}{ FAMILY CARCHARHINIDAE } \\
\hline Carcharhinus falciformis (Müller et Henle, 1839) & $\mathrm{b}$ & & $\mathrm{F}$ & $\mathrm{CT}$ \\
\hline Carcharhinus limbatus (Müller et Henle, 1839) & $\mathrm{b}$ & & $\mathrm{F}$ & $\mathrm{CT}$ \\
\hline Nasolamia velox (Gilbert, 1898) & & SIO & $\mathrm{D}$ & SP-PP \\
\hline Rhizoprionodon longurio (Jordan et Gilbert, 1882) & & CICIMAR-CI & $\mathrm{D}$ & SP-PP \\
\hline \multicolumn{5}{|l|}{ FAMILY SPHYRNIDAE } \\
\hline Sphyrna lewini (Griffith et Smith, 1834) & & 4 & $\mathrm{~F}$ & $\mathrm{CT}$ \\
\hline
\end{tabular}


Table 1 (cont.)

\begin{tabular}{|c|c|c|c|c|}
\hline Species & $\begin{array}{l}\text { Field } \\
\text { records }\end{array}$ & Record code & Habitat & $\begin{array}{l}\text { Ichthyogeographic } \\
\text { affinities }\end{array}$ \\
\hline \multicolumn{5}{|l|}{ FAMILY SQUATINIDAE } \\
\hline $\begin{array}{l}\text { Squatina californica Ayres, } 1859 \\
\text { FAMILY NARCINIDAE }\end{array}$ & a & CICIMAR-CI, 4 & $\mathrm{C}$ & AP-CP \\
\hline Diplobatis ommata (Jordan et Gilbert, 1890) & a & $\begin{array}{l}\text { CICIMAR-CI, LACM, } \\
\text { SIO, SU }\end{array}$ & $\mathrm{C}$ & SP-PP \\
\hline \multicolumn{5}{|l|}{ FAMILY RHINOBATIDAE } \\
\hline \multicolumn{5}{|l|}{ FAMILY UROTRYGONIDAE } \\
\hline $\begin{array}{l}\text { Urobatis concentricus Osburn et Nichols, } 1916 \\
\text { FAMILY UROLOPHIDAE }\end{array}$ & \multicolumn{3}{|c|}{ FAMILY UROLOPHIDAE } & SP-MP \\
\hline Urolophus halleri Cooper, 1863 & a & 4 & $\mathrm{C}$ & OP-PP \\
\hline \multicolumn{5}{|l|}{ FAMILY DASYATIDAE } \\
\hline $\begin{array}{l}\text { Dasyatis dipterura (Jordan et Gilbert, 1880) } \\
\text { FAMILY MYLIOBATIDAE }\end{array}$ & a & & $\mathrm{C}$ & SP-PCHP \\
\hline Mobula japanica (Müller et Henle, 1841) & & 4 & $\mathrm{~F}$ & $\mathrm{CT}$ \\
\hline Mobula munkiana Notarbartolo-di-Sciara, 1987 & & $\mathrm{SIO}$ & $\mathrm{F}$ & CP-PP \\
\hline Mobula thurstoni (Lloyd, 1908) & & SIO & $\mathrm{E}$ & $\mathrm{CT}$ \\
\hline Myliobatis californica Gill, 1865 & & 4 & $\mathrm{C}$ & $\mathrm{OP}-\mathrm{CP}$ \\
\hline \multicolumn{5}{|l|}{ CLASS ACTINOPTERYGII } \\
\hline \multicolumn{5}{|l|}{ FAMILY ELOPIDAE } \\
\hline Elops affinis Regan, 1909 & $\mathrm{~b}$ & & $\mathrm{E}$ & SP-PP \\
\hline \multicolumn{5}{|l|}{ FAMILY ALBULIDAE } \\
\hline Albula esuncula (Garman, 1899) & $\mathrm{b}$ & & $\mathrm{D}$ & CP-PP \\
\hline \multicolumn{5}{|l|}{ FAMILY OPHICHTHIDAE } \\
\hline Callechelys cliffi Böhlke et Briggs, 1954 & & SIO & $\mathrm{C}$ & CP-PP \\
\hline $\begin{array}{l}\text { Callechelys eristigma McCosker } \\
\text { et Rosenblatt, } 1972\end{array}$ & & $\mathrm{SIO}$ & $\mathrm{C}$ & CP-PP \\
\hline Ichthyapus selachops (Jordan et Gilbert, 1882) & & $\mathrm{SIO}$ & $\mathrm{B}$ & CP-PP \\
\hline Myrichthys tigrinus Girard, 1859 & & LACM, SIO & $\mathrm{C}$ & CP-PP \\
\hline Myrophis vafer Jordan et Gilbert, 1883 & & SIO & $\mathrm{B}$ & SP-PP \\
\hline Quassiremus nothochir (Gilbert, 1890) & & SIO, USNM & A & CP-PP \\
\hline \multicolumn{5}{|l|}{ FAMILY CONGRIDAE } \\
\hline Bathycongrus macrurus (Gilbert, 1891) & & $\mathrm{SIO}$ & $\mathrm{H}$ & CP-PP \\
\hline \multicolumn{5}{|l|}{ FAMILY NETTASTOMATIDAE } \\
\hline Facciolella equatorialis (Gilbert, 1891) & & $\mathrm{SIO}$ & G & SP-PP \\
\hline \multicolumn{5}{|l|}{ FAMILY MURAENIDAE } \\
\hline Anarchias galapagensis (Seale, 1940) & & SIO & A & CP-PP \\
\hline Echidna nebulosa (Ahl, 1789) & $\mathrm{a}$ & & A & $\mathrm{T}$ \\
\hline Echidna nocturna (Cope, 1872) & & SIO & A & CP-PP \\
\hline Gymnomuraena zebra (Shaw, 1797) & a & & A & $\mathrm{T}$ \\
\hline Gymnothorax castaneus (Jordan et Gilbert, 1883) & $a, b$ & SIO & A & CP-PP \\
\hline Gymnothorax equatorialis (Hildebrand, 1946) & $\mathrm{a}$ & & $\mathrm{B}$ & SP-PP \\
\hline Gymnothorax panamensis (Steindachner, 1876) & & CAS, LACM, SIO & A & CP-PP \\
\hline Muraena lentiginosa Jenyns, 1842 & a & LACM, SIO, USNM & A & CP-PP \\
\hline Uropterygius polystictus Myers et Wade, 1941 & & $\mathrm{SIO}$ & A & $\mathrm{CP}, \mathrm{PP}$ \\
\hline \multicolumn{5}{|l|}{ FAMILY ENGRAULIDAE } \\
\hline Anchoa exigua (Jordan et Gilbert, 1882) & & MCZ, USNM & $\mathrm{E}$ & SP-PP \\
\hline Anchoa ischana (Jordan et Gilbert, 1882) & & YPM & $\mathrm{E}$ & CP-PP \\
\hline \multicolumn{5}{|l|}{ FAMILY DUSSUMIERIIDAE } \\
\hline Etrumeus teres (DeKay, 1842) & & $\mathrm{SIO}$ & $\mathrm{E}$ & $\mathrm{CT}$ \\
\hline \multicolumn{5}{|l|}{ FAMILY CLUPEIDAE } \\
\hline Harengula thrissina (Jordan et Gilbert, 1882) & $\mathrm{a}, \mathrm{c}$ & YPM & $\mathrm{E}$ & CP-PCHP \\
\hline
\end{tabular}


Table 1 (cont.)

\begin{tabular}{|c|c|c|c|c|}
\hline Species & $\begin{array}{l}\text { Field } \\
\text { records }\end{array}$ & Record code & Habitat & $\begin{array}{l}\text { Ichthyogeographic } \\
\text { affinities }\end{array}$ \\
\hline Opisthonema libertate (Günther, 1867) & $\mathrm{c}$ & & $\mathrm{E}$ & SP-PP \\
\hline Opisthonema medirastre Berry et Barrett, 1963 & $\mathrm{c}$ & & $\mathrm{E}$ & OP-PP \\
\hline Sardinops sagax (Jenyns, 1842) & & $\mathrm{SIO}$ & $\mathrm{E}$ & AP-CP \\
\hline \multicolumn{5}{|l|}{ FAMILY CHANIDAE } \\
\hline Chanos chanos (Forsskål, 1775) & $\mathrm{b}$ & IBUNAM & $\mathrm{E}$ & $\mathrm{T}$ \\
\hline \multicolumn{5}{|l|}{ FAMILY ARIIDAE } \\
\hline Bagre panamensis (Gill, 1863) & & 4 & $\mathrm{~B}$ & SP-PP \\
\hline Occidentarius platypogon (Günther, 1864) & $\mathrm{b}$ & IBUNAM & $\mathrm{B}$ & SP-PP \\
\hline \multicolumn{5}{|l|}{ FAMILY ARGENTINIDAE } \\
\hline Argentina sialis Gilbert, 1890 & & $\mathrm{SIO}$ & $\mathrm{B}$ & OP-CP \\
\hline \multicolumn{5}{|l|}{ FAMILY AULOPIDAE } \\
\hline Aulopus bajacali Parin et Kotlyar, 1984 & & $\mathrm{SIO}$ & $\mathrm{B}$ & SP-CP \\
\hline \multicolumn{5}{|l|}{ FAMILY SYNODONTIDAE } \\
\hline Synodus evermanni Jordan et Bollman, 1890 & & $\mathrm{SIO}$ & $\mathrm{B}$ & SP-PP \\
\hline Synodus lacertinus Gilbert, 1890 & & LACM, SIO & $\mathrm{C}$ & CP-PCHP \\
\hline Synodus scituliceps Jordan et Gilbert, 1882 & & SIO, YPM & B & SP-PCHP \\
\hline \multicolumn{5}{|l|}{ FAMILY MYCTOPHIDAE } \\
\hline Benthosema panamense (Tåning, 1932) & & $\mathrm{SIO}$ & G & SP-PP \\
\hline \multicolumn{5}{|l|}{ FAMILY HOLOCENTRIDAE } \\
\hline Myripristis leiognathus Valenciennes, 1846 & $a, b$ & CAS, LACM, SIO & A & SP-PP \\
\hline Sargocentron suborbitalis (Gill, 1863) & $\mathrm{a}, \mathrm{b}, \mathrm{c}$ & $\begin{array}{l}\text { CICIMAR-CI, LACM, } \\
\text { SIO, } 1\end{array}$ & A & CP-PP \\
\hline \multicolumn{5}{|l|}{ FAMILY MUGILIDAE } \\
\hline Mugil cephalus Linnaeus, 1758 & $a, b, c$ & IBUNAM, SIO, 4 & $\mathrm{D}$ & $\mathrm{CT}$ \\
\hline \multicolumn{4}{|l|}{ FAMILY SYNGNATHIDAE } & $\mathrm{CT}$ \\
\hline Doryrhamphus excisus Kaup, 1856 & & $\begin{array}{l}\text { CAS, CICIMAR-CI, } \\
\text { SIO, }\end{array}$ & A & $\mathrm{T}$ \\
\hline \multicolumn{5}{|l|}{ FAMILY AULOSTOMIDAE } \\
\hline \multicolumn{5}{|l|}{ FAMILY FISTULARIIDAE } \\
\hline Fistularia commersonii Rüppell, 1838 & $\mathrm{a}, \mathrm{c}$ & $\begin{array}{l}\text { AMNH, IBUNAM, } \\
\text { SIO }\end{array}$ & A & $\mathrm{CT}$ \\
\hline Fistularia corneta Gilbert et Starks, 1904 & & LACM & $\mathrm{D}$ & OP-PCHP \\
\hline Atherinops affinis (Ayres, 1860) & $\mathrm{c}$ & & $\mathrm{E}$ & $\mathrm{AP}-\mathrm{CP}$ \\
\hline \multicolumn{5}{|l|}{ FAMILY BELONIDAE } \\
\hline Ablennes hians (Valenciennes, 1846) & $a, b$ & LACM, SIO & $\mathrm{F}$ & $\mathrm{CT}$ \\
\hline Platybelone argalus (Lesueur, 1821) & & SIO & $\mathrm{E}$ & $\mathrm{CT}$ \\
\hline Strongylura exilis (Girard, 1854) & & LACM, SIO, 1 & $\mathrm{E}$ & OP-PP \\
\hline Tylosurus pacificus (Steindachner, 1876) & $\mathrm{a}, \mathrm{b}, \mathrm{c}$ & SIO & $\mathrm{F}$ & SP-PP \\
\hline \multicolumn{5}{|l|}{ FAMILY EXOCOETIDAE } \\
\hline Fodiator acutus (Valenciennes, 1847) & & SIO & $\mathrm{E}$ & SP-PCHP \\
\hline \multicolumn{5}{|l|}{ FAMILY HEMIRAMPHIDAE } \\
\hline Hemiramphus saltator Gilbert et Starks, 1904 & $\mathrm{a}, \mathrm{c}$ & LACM & $\mathrm{E}$ & CP-PP \\
\hline \multicolumn{5}{|l|}{ FAMILY ZANCLIDAE } \\
\hline Zanclus cornutus (Linnaeus, 1758) & $\mathrm{a}$ & & A & $\mathrm{T}$ \\
\hline \multicolumn{5}{|l|}{ FAMILY ACANTHURIDAE } \\
\hline Acanthurus nigricans (Linnaeus, 1758) & $\mathrm{a}$ & & A & $\mathrm{T}$ \\
\hline Acanthurus triostegus (Linnaeus, 1758) & $\mathrm{a}$ & & $\mathrm{A}$ & $\mathrm{T}$ \\
\hline Acanthurus xanthopterus Valenciennes, 1835 & $\mathrm{a}$ & 1 & A & $\mathrm{T}$ \\
\hline Prionurus punctatus Gill, 1862 & $\mathrm{a}$ & 1 & A & CP-PP \\
\hline
\end{tabular}


Table 1 (cont.)

Species
Field Record code

Porichthys analis Hubbs et Schultz, 1939

FAMILY TRIPTERYGIIDAE

Enneanectes carminalis (Jordan et Gilbert, 1882)

Axoclinus lucillae Fowler, 1944

Axoclinus nigricaudus Allen et Robertson, 1991

Axoclinus sp.

Crocodilichthys gracilis Allen et Robertson, 1991

Enneanectes reticulatus Allen et Robertson, 1991

FAMILY DACTYLOSCOPIDAE

Dactylagnus mundus Gill, 1863

Dactyloscopus lunaticus Gilbert, 1890

Dactyloscopus pectoralis Gill, 1861

Gillellus semicinctus Gilbert, 1890

FAMILY BLENNIIDAE

Hypsoblennius brevipinnis (Günther, 1861)

Hypsoblennius gentilis (Girard, 1854)

Ophioblennius steindachneri Jordan

et Evermann, 1898

Plagiotremus azaleus (Jordan et Bollman, 1890)

FAMILY CHAENOPSIDAE

Acanthemblemaria crockeri Beebe et Tee-Van, 1938

Acanthemblemaria hastingsi Lin et Galland, 2010

Chaenopsis alepidota (Gilbert, 1890)

Cirriemblemaria lucasana (Stephens, 1963)

Coralliozetus angelicus (Böhlke et Mead, 1957)

Coralliozetus micropes (Beebe et Tee-Van, 1938)

Coralliozetus rosenblatti Stephens, 1963

Emblemaria hypacanthus

(Jenkins et Evermann, 1889)

Emblemaria walkeri Stephens, 1963

Protemblemaria bicirrus (Hildebrand, 1946)

Stathmonotus sinuscalifornici (Chabanaud, 1942)

FAMILY LABRISOMIDAE

Labrisomus striatus Hubbs, 1953

Labrisomus xanti Gill, 1860

Malacoctenus hubbsi Springer, 1959

Malacoctenus mexicanus Springer, 1959

Malacoctenus tetranemus (Cope, 1877)

Paraclinus sini Hubbs, 1952

Starksia cremnobates (Gilbert, 1890)

Starksia spinipenis (Al-Uthman, 1960)

Xenomedea rhodopyga Rosenblatt et Taylor, 1971

FAMILY NEMATISTIIDAE

Nematistius pectoralis Gill, 1862

FAMILY CORYPHAENIDAE

Coryphaena hippurus Linnaeus, 1758

FAMILY CARANGIDAE

Caranx caballus Günther, 1868

Caranx caninus Günther, 1867

\begin{tabular}{|c|c|c|c|}
\hline & SIO & B & CP-MP \\
\hline \multirow{6}{*}{$\mathrm{a}$} & LACM, SIO & A & SP-PP \\
\hline & & A & MP-PP \\
\hline & SIO & A & $\mathrm{CP}$ \\
\hline & & A & \\
\hline & SIO, USNM, 1 & A & $\mathrm{CP}$ \\
\hline & IBUNAM, SIO & A & SP-CP \\
\hline \multirow{4}{*}{$\mathrm{c}$} & $\begin{array}{l}\text { IBUNAM, SIO, } \\
\text { USNM }\end{array}$ & B & SP-PP \\
\hline & AMNH & B & CP-PP \\
\hline & SIO & B & $\mathrm{CP}$ \\
\hline & LACM & B & SP-PP \\
\hline \multirow{4}{*}{$\mathrm{a}$} & SIO & A & SP-PP \\
\hline & SIO & A & $\mathrm{OP}-\mathrm{CP}$ \\
\hline & $\begin{array}{l}\text { CICIMAR-CI, } \\
\text { IBUNAM, LACM, } \\
\text { SIO, } 1\end{array}$ & A & SP-PP \\
\hline & CAS, SIO & A & SP-PP \\
\hline \multirow{6}{*}{$\mathrm{c}$} & CAS, IBUNAM, SIO & A & $\mathrm{CP}$ \\
\hline & SIO & A & $\mathrm{CP}$ \\
\hline & $\begin{array}{l}\text { ANSP, IBUNAM, } \\
\text { SIO, SU }\end{array}$ & A & SP-CP \\
\hline & SIO & A & CP-MP \\
\hline & IBUNAM, SIO & A & CP-MP \\
\hline & SIO & A & $\mathrm{CP}$ \\
\hline \multirow{14}{*}{$\mathrm{a}$} & SIO & A & $\mathrm{CP}$ \\
\hline & $\begin{array}{l}\text { AMNH, IBUNAM, } \\
\text { SIO }\end{array}$ & A & $\mathrm{CP}$ \\
\hline & IBUNAM & A & $\mathrm{CP}$ \\
\hline & SIO & A & CP-PP \\
\hline & SIO & A & $\mathrm{CP}$ \\
\hline & SIO & $\mathrm{A}$ & CP-MP \\
\hline & LACM, SIO & A & SP-MP \\
\hline & $\begin{array}{l}\text { IBUNAM, LACM, } \\
\text { SIO }\end{array}$ & A & $\mathrm{CP}$ \\
\hline & LACM, SIO & A & CP-MP \\
\hline & LACM, SIO & A & CP-PP \\
\hline & IBUNAM, SIO & A & SP-CP \\
\hline & SIO & A & $\mathrm{CP}$ \\
\hline & SIO & A & CP-MP \\
\hline & SIO, & A & $\mathrm{CP}$ \\
\hline $\mathrm{b}$ & AMNH & $\mathrm{E}$ & SP-PP \\
\hline $\mathrm{a}$ & & $\mathrm{F}$ & $\mathrm{CT}$ \\
\hline $\mathrm{a}, \mathrm{b}$ & CICIMAR-CI & $\mathrm{E}$ & OP-PCHP \\
\hline $\mathrm{a}, \mathrm{b}, \mathrm{c}$ & & $\mathrm{E}$ & SP-PCHP \\
\hline
\end{tabular}


Table 1 (cont.)

\begin{tabular}{|c|c|c|c|c|}
\hline Species & $\begin{array}{l}\text { Field } \\
\text { records }\end{array}$ & Record code & Habitat & $\begin{array}{l}\text { Ichthyogeographic } \\
\text { affinities }\end{array}$ \\
\hline Caranx sexfasciatus Quoy et Gaimard, 1825 & $\mathrm{a}, \mathrm{b}$ & & $\mathrm{E}$ & $\mathrm{T}$ \\
\hline Decapterus macarellus (Cuvier, 1833) & $\mathrm{a}, \mathrm{b}$ & & $\mathrm{F}$ & $\mathrm{CT}$ \\
\hline Elagatis bipinnulata (Quoy et Gaimard, 1825) & & CICIMAR-CI & $\mathrm{F}$ & $\mathrm{CT}$ \\
\hline Gnathanodon speciosus (Forsskål, 1775) & $\mathrm{a}$ & & $\mathrm{E}$ & $\mathrm{T}$ \\
\hline Naucrates ductor (Linnaeus, 1758) & $\mathrm{a}$ & & $\mathrm{F}$ & $\mathrm{CT}$ \\
\hline Selar crumenophthalmus (Bloch, 1793) & & $\mathrm{MCZ}$ & $\mathrm{E}$ & $\mathrm{CT}$ \\
\hline Selene brevoortii (Gill, 1863) & $\mathrm{b}$ & & $\mathrm{D}$ & SP-PCHP \\
\hline Selene peruviana (Guichenot, 1866) & $\mathrm{a}$ & & $\mathrm{D}$ & SP-PCHP \\
\hline Seriola lalandi Valenciennes, 1833 & $\mathrm{a}$ & & $\mathrm{D}$ & $\mathrm{CT}$ \\
\hline Seriola peruana Steindachner, 1881 & & 3 & $\mathrm{D}$ & CP-PP \\
\hline Seriola rivoliana Valenciennes, 1833 & $\mathrm{a}$ & & $\mathrm{D}$ & $\mathrm{CT}$ \\
\hline Trachinotus rhodopus Gill, 1863 & $\mathrm{a}, \mathrm{b}, \mathrm{c}$ & & $\mathrm{D}$ & SP-PCHP \\
\hline \multicolumn{5}{|l|}{ FAMILY GOBIESOCIDAE } \\
\hline Arcos erythrops (Jordan et Gilbert, 1882) & & LACM & A & CP-MP \\
\hline Tomicodon boehlkei Briggs, 1955 & & IBUNAM, LACM & A & $\mathrm{CP}$ \\
\hline Tomicodon humeralis (Gilbert, 1890) & & IBUNAM & A & $\mathrm{CP}$ \\
\hline Tomicodon myersi Briggs, 1955 & & $\mathrm{SIO}$ & A & CP-PP \\
\hline \multicolumn{5}{|l|}{ FAMILY GOBIIDAE } \\
\hline Aruma histrio (Jordan, 1884) & & $\mathrm{SIO}$ & A & $\mathrm{CP}$ \\
\hline Barbulifer pantherinus (Pellegrin, 1901) & & $\mathrm{SIO}$ & A & $\mathrm{CP}$ \\
\hline Bathygobius ramosus Ginsburg, 1947 & & IBUNAM & A & CP-PP \\
\hline Chriolepis cuneata Bussing, 1990 & & $\mathrm{SIO}$ & A & CP-PP \\
\hline Chriolepis zebra Ginsburg, 1938 & & $\mathrm{SIO}$ & $\mathrm{C}$ & $\mathrm{CP}$ \\
\hline Coryphopterus urospilus Ginsburg, 1938 & & LACM, SIO, 1 & $\mathrm{C}$ & CP-PP \\
\hline Ctenogobius sagittula (Günther, 1862) & $\mathrm{c}$ & & $\mathrm{B}$ & SP-PP \\
\hline Elacatinus limbaughi Hoese et Reader, 2001 & & $\mathrm{SIO}$ & A & $\mathrm{CP}$ \\
\hline Elacatinus punticulatus (Ginsburg, 1938) & $\mathrm{a}$ & SIO, 1 & A & CP-PP \\
\hline Gobiosoma chiquita (Jenkins et Evermann, 1889) & $\mathrm{c}$ & IBUNAM, SIO & $\mathrm{C}$ & $\mathrm{CP}$ \\
\hline Gymneleotris seminuda (Günther, 1864) & & $\mathrm{SIO}$ & A & CP-PP \\
\hline Lythrypnus dalli (Gilbert, 1890) & & LACM, SIO, SU, 1 & A & SP-PP \\
\hline Lythrypnus pulchellus Ginsburg, 1938 & & $\mathrm{SIO}$ & A & CP-PP \\
\hline Pycnomma semisquamatum Rutter, 1904 & & $\mathrm{SIO}$ & A & $\mathrm{CP}$ \\
\hline Quietula y-cauda (Jenkins et Evermann, 1889) & & SU & $\mathrm{B}$ & $\mathrm{OP}-\mathrm{CP}$ \\
\hline \multicolumn{5}{|l|}{ FAMILY LABRIDAE } \\
\hline Bodianus diplotaenia (Gill, 1862) & $\mathrm{a}$ & $\mathrm{SIO}, 1$ & A & SP-PCHP \\
\hline Decodon melasma Gomon, 1974 & & $\mathrm{SIO}$ & $\mathrm{C}$ & SP-PP \\
\hline Halichoeres chierchiae Di Caporiacco, 1948 & $\mathrm{a}$ & 1 & A & CP-PP \\
\hline Halichoeres dispilus (Günther, 1864) & $\mathrm{a}$ & $\mathrm{SIO}, 1$ & A & SP-PP \\
\hline Halichoeres melanotis (Gilbert, 1890) & $\mathrm{a}$ & & A & SP-PP \\
\hline Halichoeres nicholsi (Jordan et Gilbert, 1882) & $\mathrm{a}$ & 1 & A & CP-PP \\
\hline Halichoeres notospilus (Günther, 1864) & $\mathrm{a}$ & & A & CP-PP \\
\hline Halichoeres semicinctus (Ayres, 1859) & $\mathrm{a}$ & & A & SP-CP \\
\hline Iniistius pavo (Valenciennes, 1840) & & 1 & A & $\mathrm{T}$ \\
\hline Novaculichthys taeniourus (Lacepède, 1801) & $\mathrm{a}$ & & A & $\mathrm{T}$ \\
\hline Thalassoma grammaticum Gilbert, 1890 & $\mathrm{a}$ & & A & CP-PP \\
\hline Thalassoma lucasanum (Gill, 1862) & $\mathrm{a}, \mathrm{c}$ & $\begin{array}{l}\text { BPBM, CAS, LACM, } \\
\text { SIO, YPM, } 1\end{array}$ & A & SP-PP \\
\hline \multicolumn{5}{|l|}{ FAMILY SCARIDAE } \\
\hline $\begin{array}{l}\text { Nicholsina denticulata } \\
\text { (Evermann et Radcliffe, 1917) }\end{array}$ & $a, b$ & LACM, SIO & A & SP-PP \\
\hline Scarus compressus (Osburn et Nichols, 1916) & $\mathrm{a}, \mathrm{b}$ & 1 & A & CP-PP \\
\hline Scarus ghobban Forsskål, 1775 & $\mathrm{a}, \mathrm{b}$ & AMNH, SIO, 4, 1 & A & $\mathrm{T}$ \\
\hline Scarus perrico Jordan et Gilbert, 1882 & $\mathrm{a}$ & AMNH, 1 & A & CP-PP \\
\hline
\end{tabular}


Table 1 (cont.)

\begin{tabular}{|c|c|c|c|c|}
\hline Species & $\begin{array}{l}\text { Field } \\
\text { records }\end{array}$ & Record code & Habitat & $\begin{array}{l}\text { Ichthyogeographic } \\
\text { affinities }\end{array}$ \\
\hline Scarus rubroviolaceus Bleeker, 1847 & $\mathrm{a}, \mathrm{b}$ & 1 & $\mathrm{~A}$ & $\mathrm{~T}$ \\
\hline \multicolumn{5}{|l|}{ FAMILY POMACENTRIDAE } \\
\hline Abudefduf declivifrons (Gill, 1862) & $\mathrm{a}$ & & A & CP-PP \\
\hline Abudefduf troschelii (Gill, 1862) & $\mathrm{a}, \mathrm{b}, \mathrm{c}$ & $\begin{array}{l}\text { IBUNAM, LACM, } \\
\text { SIO, } 1\end{array}$ & A & SP-PP \\
\hline Chromis atrilobata Gill, 1862 & $\mathrm{a}$ & CAS, LACM, SIO, 1 & A & SP-PCHP \\
\hline Chromis limbaughi Greenfield et Woods, 1980 & & SIO & A & CP-MP \\
\hline Microspathodon bairdii (Gill, 1862) & $\mathrm{a}$ & & $\mathrm{A}$ & CP-PP \\
\hline Microspathodon dorsalis (Gill, 1862) & $\mathrm{a}$ & AMNH, LACM, SIO, 1 & A & CP-PP \\
\hline Stegastes acapulcoensis (Fowler, 1944) & $\mathrm{a}$ & & A & CP-PP \\
\hline Stegastes flavilatus (Gill, 1862) & $\mathrm{a}$ & 1 & A & SP-PP \\
\hline Stegastes leucorus (Gilbert, 1892) & $\mathrm{a}$ & & A & SP-MP \\
\hline Stegastes rectifraenum (Gill, 1862) & $\mathrm{a}$ & $\begin{array}{l}\text { AMNH, CAS, LACM, } \\
\text { SIO, } 1\end{array}$ & A & SP-MP \\
\hline \multicolumn{5}{|l|}{ FAMILY LOPHIIDAE } \\
\hline Lophiodes spilurus (Garman, 1899) & & $\mathrm{SIO}$ & $\mathrm{B}$ & OP-PP \\
\hline \multicolumn{5}{|l|}{ FAMILY ANTENNARIIDAE } \\
\hline Fowlerichthys avalonis (Jordan et Starks, 1907) & & SIO & $\mathrm{C}$ & SP-PCHP \\
\hline Antennatus sanguineus (Gill, 1863) & & $\mathrm{SIO}$ & A & CP-PP \\
\hline Antennatus strigatus (Gill, 1863) & & $\mathrm{SIO}$ & $\mathrm{A}$ & CP-PP \\
\hline \multicolumn{5}{|l|}{ FAMILY CARAPODIDAE } \\
\hline Echiodon exsilium Rosenblatt, 1961 & & $\mathrm{SIO}$ & $\mathrm{C}$ & CP-PP \\
\hline Carapus dubius (Putnam, 1874) & & CAS & $\mathrm{C}$ & CP-PP \\
\hline \multicolumn{5}{|l|}{ FAMILY OPHIDIIDAE } \\
\hline Cherublemma emmelas (Gilbert, 1890) & & UMMZ & $\mathrm{B}$ & CP-PCHP \\
\hline Lepophidium microlepis (Gilbert, 1890) & & $\mathrm{SIO}$ & $\mathrm{B}$ & CP, PP \\
\hline Neobythites stelliferoides Gilbert, 1890 & & $\mathrm{SIO}$ & $\mathrm{B}$ & CP-PP \\
\hline Ophidion iris Breder, 1936 & & SIO & $\mathrm{B}$ & $\mathrm{CP}$ \\
\hline Otophidium indefatigabile Jordan et Bollman, 1890 & & $\mathrm{SIO}$ & $\mathrm{B}$ & CP-PP \\
\hline \multicolumn{5}{|l|}{ FAMILY BYTHITIDAE } \\
\hline $\begin{array}{l}\text { Grammonus diagrammus } \\
\text { (Heller et Snodgrass, 1903) }\end{array}$ & & CAS, SIO & A & SP-PP \\
\hline \multicolumn{5}{|l|}{ FAMILY OPISTOGNATHIDAE } \\
\hline Opistognathus brochus Bussing et Lavenberg, 2003 & & SIO & $\mathrm{C}$ & $\mathrm{CP}, \mathrm{PP}$ \\
\hline Opistognathus fossoris Bussing et Lavenberg, 2003 & & $\mathrm{SIO}$ & $\mathrm{C}$ & $\mathrm{CP}$ \\
\hline Opistognathus punctatus Peters, 1869 & $\mathrm{a}$ & CICIMAR-CI, SIO & $\mathrm{C}$ & SP-PP \\
\hline Opistognathus rhomaleus Jordan et Gilbert, 1881 & & LACM, SIO & $\mathrm{C}$ & SP-PP \\
\hline Opistognathus rosenblatti Allen et Robertson, 1991 & & $\mathrm{SIO}$ & $\mathrm{C}$ & SP-CP \\
\hline \multicolumn{5}{|l|}{ FAMILY APOGONIDAE } \\
\hline Apogon pacificus (Herre, 1935) & $\mathrm{a}$ & 1 & A & SP-PCHP \\
\hline Apogon retrosella (Gill, 1862) & $\mathrm{a}$ & $\begin{array}{l}\text { AMNH, LACM, SIO, } \\
\text { SU, } 1\end{array}$ & A & SP-PP \\
\hline \multicolumn{5}{|l|}{ FAMILY MALACANTHIDAE } \\
\hline Caulolatilus affinis Gill, 1865 & & $\mathrm{SIO}$ & $\mathrm{D}$ & SP-PCHP \\
\hline \multicolumn{5}{|l|}{ FAMILY LUTJANIDAE } \\
\hline Hoplopagrus guentherii Gill, 1862 & $a, b, c$ & AMNH, SIO, 4 & $\mathrm{D}$ & SP-PP \\
\hline Lutjanus aratus (Günther, 1864) & $a, b$ & & $\mathrm{D}$ & CP-PP \\
\hline Lutjanus argentiventris (Peters, 1869) & $\mathrm{a}, \mathrm{b}, \mathrm{c}$ & IBUNAM, SIO, 4, 1 & $\mathrm{D}$ & SP-PCHP \\
\hline Lutjanus colorado Jordan et Gilbert, 1882 & $\mathrm{a}$ & & $\mathrm{D}$ & SP-PP \\
\hline Lutjanus guttatus (Steindachner, 1869) & $\mathrm{b}$ & & $\mathrm{D}$ & CP-PCHP \\
\hline Lutjanus jordani (Gilbert, 1898) & & YPM & $\mathrm{D}$ & SP-PP \\
\hline Lutjanus novemfasciatus Gill, 1862 & $\mathrm{a}, \mathrm{b}$ & $\begin{array}{l}\text { AMNH, IBUNAM, } \\
\text { SIO }\end{array}$ & $\mathrm{D}$ & SP-PP \\
\hline Lutjanus peru (Nichols et Murphy, 1922) & $\mathrm{a}$ & & $\mathrm{D}$ & SP-PCHP \\
\hline
\end{tabular}


Table 1 (cont.)

\begin{tabular}{|c|c|c|c|c|}
\hline Species & $\begin{array}{l}\text { Field } \\
\text { records }\end{array}$ & Record code & Habitat & $\begin{array}{l}\text { Ichthyogeographic } \\
\text { affinities }\end{array}$ \\
\hline Lutjanus viridis (Valenciennes, 1846) & $\mathrm{a}$ & & A & CP-PP \\
\hline \multicolumn{5}{|l|}{ FAMILY GERREIDAE } \\
\hline Eucinostomus currani Zahuranec, 1980 & $\mathrm{a}, \mathrm{c}$ & & $\mathrm{B}$ & SP-PCHP \\
\hline Eucinostomus dowii (Gill, 1863) & $\mathrm{b}, \mathrm{c}$ & $\begin{array}{l}\text { IBUNAM, SIO, } \\
\text { USNM }\end{array}$ & $\mathrm{B}$ & SP-PP \\
\hline Eucinostomus entomelas Zahuranec, 1980 & $\mathrm{c}$ & SIO & $\mathrm{B}$ & SP-PP \\
\hline Eucinostomus gracilis (Gill, 1862) & $\mathrm{c}$ & & $\mathrm{B}$ & SP-PP \\
\hline Gerres cinereus (Walbaum, 1792) & $\mathrm{a}, \mathrm{b}, \mathrm{c}$ & CICIMAR-CI, SIO, 4 & $\mathrm{~B}$ & $\mathrm{AM}$ \\
\hline \multicolumn{5}{|l|}{ FAMILY HAEMULIDAE } \\
\hline Anisotremus interruptus (Gill, 1862) & $a, b, c$ & $\begin{array}{l}\text { AMNH, IBUNAM, } \\
\text { SIO }\end{array}$ & $\mathrm{D}$ & SP-PP \\
\hline Anisotremus taeniatus Gill, 1861 & $\mathrm{a}$ & & A & CP-PP \\
\hline Haemulon flaviguttatum Gill, 1862 & $a, b$ & IBUNAM, SIO & $\mathrm{D}$ & SP-PP \\
\hline Haemulon maculicauda (Gill, 1862) & $a, b$ & SIO & $\mathrm{D}$ & CP-PP \\
\hline Haemulon scudderii Gill, 1862 & $\mathrm{a}, \mathrm{b}$ & CICIMAR-CI & $\mathrm{D}$ & CP-PP \\
\hline Haemulon sexfasciatum Gill, 1862 & $\mathrm{a}, \mathrm{b}$ & $\begin{array}{l}\text { AMNH, CICIMAR-CI, } \\
\text { IBUNAM, SIO, } 4,1\end{array}$ & $\mathrm{D}$ & CP-PP \\
\hline Haemulon steindachneri (Jordan et Gilbert, 1882) & $a, b$ & SIO & $\mathrm{D}$ & $\mathrm{AM}$ \\
\hline Microlepidotus inornatus Gill, 1862 & $\mathrm{a}, \mathrm{b}$ & $\begin{array}{l}\text { IBUNAM, LACM, } \\
\text { SIO }\end{array}$ & $\mathrm{D}$ & SP-MP \\
\hline Orthopristis chalceus (Günther, 1864) & $\mathrm{b}, \mathrm{c}$ & & $\mathrm{D}$ & SP-PCHP \\
\hline Pomadasys panamensis (Steindachner, 1876) & $\mathrm{b}$ & & $\mathrm{D}$ & CP-PP \\
\hline Xenichthys xanti Gill, 1863 & $\mathrm{a}$ & & $\mathrm{D}$ & CP-PCHP \\
\hline Xenistius californiensis (Steindachner, 1876) & & $\mathrm{SIO}$ & $\mathrm{D}$ & OP-PP \\
\hline \multicolumn{5}{|l|}{ FAMILY SPARIDAE } \\
\hline Calamus brachysomus (Lockington, 1880) & $\mathrm{a}$ & & $\mathrm{C}$ & SP-PCHP \\
\hline \multicolumn{5}{|l|}{ FAMILY POLYNEMIDAE } \\
\hline Polydactylus approximans (Lay et Bennett, 1839) & $\mathrm{c}$ & & $\mathrm{B}$ & OP-PCHP \\
\hline \multicolumn{5}{|l|}{ FAMILY SCIAENIDAE } \\
\hline Pareques viola (Gilbert, 1898) & $\mathrm{a}$ & AMNH, LACM, SIO & A & $\mathrm{CP}, \mathrm{PP}$ \\
\hline Umbrina analis Günther, 1868 & $\mathrm{c}$ & & $\mathrm{D}$ & CP-PP \\
\hline Umbrina xanti Gill, 1862 & & IBUNAM & $\mathrm{B}$ & CP-PCHP \\
\hline \multicolumn{5}{|l|}{ FAMILY MULLIDAE } \\
\hline Mulloidichthys dentatus (Gill, 1862) & $\mathrm{a}, \mathrm{b}$ & $\begin{array}{l}\text { AMNH, CICIMAR-CI, } \\
\text { IBUNAM, SIO, } 4,1\end{array}$ & $\mathrm{C}$ & CP-PP \\
\hline Pseudupeneus grandisquamis (Gill, 1863) & & IBUNAM & $\mathrm{B}$ & SP-PCHP \\
\hline \multicolumn{5}{|l|}{ FAMILY KYPHOSIDAE } \\
\hline Girella simplicidens Osburn et Nichols, 1916 & $\mathrm{a}$ & $\mathrm{SIO}$ & A & $\mathrm{CP}$ \\
\hline Kyphosus analogus (Gill, 1862) & $\mathrm{a}, \mathrm{b}$ & $\begin{array}{l}\text { AMNH, IBUNAM, } \\
\text { SIO, } 4\end{array}$ & A & SP-PP \\
\hline Kyphosus elegans (Peters, 1869) & $a, b$ & 1 & A & CP-PP \\
\hline \multicolumn{5}{|l|}{ FAMILY EPHIPPIDAE } \\
\hline Chaetodipterus zonatus (Girard, 1858) & $\mathrm{a}$ & & $\mathrm{D}$ & SP-PP \\
\hline \multicolumn{5}{|l|}{ FAMILY CHAETODONTIDAE } \\
\hline Chaetodon humeralis Günther, 1860 & $\mathrm{a}$ & CICIMAR-CI & $\mathrm{C}$ & SP-PCHP \\
\hline Johnrandallia nigrirostris $(\mathrm{Gill}, 1862)$ & $\mathrm{a}$ & $\mathrm{SIO}, 1$ & A & CP-PCHP \\
\hline \multicolumn{5}{|l|}{ FAMILY POMACANTHIDAE } \\
\hline Holacanthus clarionensis Gilbert, 1891 & $\mathrm{a}$ & & A & SP-CP \\
\hline Holacanthus passer Valenciennes, 1846 & a & $\begin{array}{l}\text { CICIMAR-CI, } \\
\text { IBUNAM, SIO, } 1\end{array}$ & A & SP-PP \\
\hline Pomacanthus zonipectus (Gill, 1862) & $\mathrm{a}$ & IBUNAM, SIO, 1 & A & SP-PP \\
\hline \multicolumn{5}{|l|}{ FAMILY CIRRHIRIDAE } \\
\hline Cirrhitichthys oxycephalus (Bleeker, 1855) & $\mathrm{a}$ & $\begin{array}{l}\text { BPBM, CAS, LACM, } \\
\text { SIO, } 1\end{array}$ & A & $\mathrm{T}$ \\
\hline
\end{tabular}


Table 1 (cont.)

\begin{tabular}{|c|c|c|c|c|}
\hline Species & $\begin{array}{l}\text { Field } \\
\text { records }\end{array}$ & Record code & Habitat & $\begin{array}{l}\text { Ichthyogeographic } \\
\text { affinities }\end{array}$ \\
\hline Cirrhitus rivulatus Valenciennes, 1846 & $\mathrm{a}$ & $\begin{array}{l}\text { IBUNAM, LACM, } \\
\text { SIO, } 1\end{array}$ & A & CP-PP \\
\hline Oxycirrhites typus Bleeker, 1857 & & CAS & A & $\mathrm{T}$ \\
\hline \multicolumn{5}{|l|}{ FAMILY BOTHIDAE } \\
\hline Bothus leopardinus (Günther, 1862) & & YPM & $\mathrm{B}$ & SP-PP \\
\hline Perissias taeniopterus (Gilbert, 1890) & & $\mathrm{SIO}, \mathrm{SU}$ & $\mathrm{C}$ & SP-PP \\
\hline \multicolumn{5}{|l|}{ FAMILY PARALICHTHYIDAE } \\
\hline Citharichthys gilberti Jenkins et Evermann, 1889 & $\mathrm{c}$ & & B & SP-PP \\
\hline Etropus crossotus Jordan et Gilbert, 1882 & $\mathrm{c}$ & & B & $\mathrm{AM}$ \\
\hline Hippoglossina bollmani Gilbert, 1890 & & $\mathrm{SIO}$ & B & SP-PP \\
\hline Syacium latifrons (Jordan et Gilbert, 1882) & & $\mathrm{SIO}$ & B & CP-PP \\
\hline Syacium ovale (Günther, 1864) & & SU & $\mathrm{B}$ & CP-PP \\
\hline \multicolumn{5}{|l|}{ FAMILY CYNOGLOSSIDAE } \\
\hline Symphurus atricaudus (Jordan et Gilbert, 1880) & & SU & B & OP-PP \\
\hline
\end{tabular}

Sphyraena lucasana Gill, 1863

$\begin{array}{llll}\text { a, b } & \text { SIO, } 1 & \text { E } & \text { SP-MP }\end{array}$

FAMILY TRICHIURIDAE

Lepidopus fitchi Rosenblatt et Wilson, 1987

FAMILY SCOMBRIDAE

Auxis rochei (Risso, 1810)

Auxis thazard eurydorax (Lacepède, 1800)

Euthynnus lineatus Kishinouye, 1920

Scomber japonicus Houttuyn, 1782

Scomberomorus sierra Jordan et Starks, 1895

Thunnus albacares (Bonnaterre, 1788)

$\mathrm{SIO}$

I OP-PP

b

b

$\mathrm{a}, \mathrm{b} \quad \mathrm{SIO}$

b $\quad$ SIO, 4

a, b

FAMILY SCORPAENIDAE

Pontinus sierra (Gilbert, 1890)

Scorpaena histrio Jenyns, 1840

Scorpaena mystes Jordan et Starks, 1895

Scorpaena sonorae Jenkins et Evermann, 1889

Scorpaenodes xyris (Jordan et Gilbert, 1882)

FAMILY TRIGLIDAE

Bellator loxias (Jordan, 1897)

Prionotus albirostris Jordan et Bollman, 1890

FAMILY SERRANIDAE

Diplectrum labarum Rosenblatt et Johnson, 1974

Diplectrum pacificum Meek et Hildebrand, 1925

Diplectrum rostrum Bortone, 1974

Paralabrax auroguttatus Walford, 1936

Paralabrax maculatofasciatus (Steindachner, 1868) a, b

Serranus aequidens Gilbert, 1890

Serranus psittacinus Valenciennes, 1846

a

Hemanthias signifer (Garman, 1899)

Pronotogrammus eos Gilbert, 1890

Alphestes immaculatus Breder, 1936

Alphestes multiguttatus (Günther, 1867)

Cephalopholis colonus (Valenciennes, 1846)

Cephalopholis panamensis (Steindachner, 1877)

b

$\mathrm{a}, \mathrm{b}, \mathrm{c}$

SIO

SIO

IBUNAM, LACM, SIO, SU

SIO

LACM, SIO

SIO

SIO

SIO

a

Dermatolepis dermatolepis (Boulenger, 1895)

Epinephelus analogus Gill, 1863

Epinephelus labriformis (Jenyns, 1840) $\mathrm{a}, \mathrm{b}$

$\mathrm{a}, \mathrm{b}$

$\mathrm{a}, \mathrm{b}$

a

a

a

\begin{tabular}{|c|c|c|}
\hline \multirow[t]{2}{*}{$\mathrm{SIO}$} & B & SP-PP \\
\hline & B & SP-PP \\
\hline $\mathrm{SIO}$ & $\mathrm{B}$ & SP-PP \\
\hline $\mathrm{SIO}$ & A & SP-MP \\
\hline $\begin{array}{l}\text { CICIMAR-CI, } \\
\text { IBUNAM }\end{array}$ & $\mathrm{D}$ & OP-MP \\
\hline $\mathrm{SIO}$ & $\mathrm{B}$ & SP-PP \\
\hline LACM, SIO, SU & A & SP-PCHP \\
\hline CICIMAR-CI & $\mathrm{D}$ & SP-PP \\
\hline $\mathrm{SIO}$ & $\mathrm{D}$ & CP-PP \\
\hline \multirow[t]{2}{*}{$\mathrm{SIO}$} & $\mathrm{C}$ & CP-PP \\
\hline & $\mathrm{C}$ & CP-PP \\
\hline LACM, SIO, 4, 1 & A & SP-PP \\
\hline LACM, SIO, 1 & A & CP-PP \\
\hline \multirow[t]{2}{*}{$\mathrm{SIO}$} & $\mathrm{A}$ & SP-PP \\
\hline & $\mathrm{C}$ & SP-PCHP \\
\hline LACM, SIO, SU, 1 & $\mathrm{~A}$ & SP-PP \\
\hline
\end{tabular}


Table 1 (cont.)

\begin{tabular}{|c|c|c|c|c|}
\hline Species & $\begin{array}{l}\text { Field } \\
\text { records }\end{array}$ & Record code & Habitat & $\begin{array}{l}\text { Ichthyogeographic } \\
\text { affinities }\end{array}$ \\
\hline Epinephelus labriformis (Jenyns, 1840) & $\mathrm{a}$ & LACM, SIO, SU, 1 & A & SP-PP \\
\hline Hyporthodus acanthistius (Gilbert, 1892) & & CAS & $\mathrm{C}$ & SP-PP \\
\hline Mycteroperca jordani (Jenkins et Evermann, 1889) & $\mathrm{a}$ & & A & SP-CP \\
\hline $\begin{array}{l}\text { Mycteroperca prionura Rosenblatt } \\
\text { et Zahuranec, } 1967\end{array}$ & & SIO & A & CP-MP \\
\hline Mycteroperca rosacea (Streets, 1877) & $\mathrm{a}, \mathrm{b}$ & $\begin{array}{l}\text { AMNH, CAS, LACM, } \\
\text { SIO, } 1\end{array}$ & A & SP-MP \\
\hline Pseudogramma thaumasia (Gilbert, 1900) & & SIO & A & CP-PP \\
\hline Rypticus bicolor Valenciennes, 1846 & $\mathrm{a}$ & CAS, LACM, SIO & A & CP-PP \\
\hline Rypticus nigripinnis Gill, 1861 & $\mathrm{a}$ & SIO & $\mathrm{C}$ & CP-PP \\
\hline \multicolumn{5}{|l|}{ FAMILY STROMATEIDAE } \\
\hline Peprilus medius (Peters, 1869) & & CICIMAR-CI & $\mathrm{E}$ & CP-PCHP \\
\hline \multicolumn{5}{|l|}{ FAMILY BALISTIDAE } \\
\hline Balistes polylepis Steindachner, 1876 & $\mathrm{a}, \mathrm{b}$ & IBUNAM, 4 & $\mathrm{D}$ & AP-PCHP \\
\hline Pseudobalistes naufragium (Jordan et Starks, 1895) & $\mathrm{a}$ & 1 & $\mathrm{D}$ & CP-PCHP \\
\hline Sufflamen verres (Gilbert et Starks, 1904) & $\mathrm{a}$ & $\begin{array}{l}\text { AMNH, IBUNAM, } \\
\text { LACM, SIO, } 1\end{array}$ & A & SP-PP \\
\hline \multicolumn{5}{|l|}{ FAMILY OSTRACIIDAE } \\
\hline Ostracion meleagris Shaw, 1796 & a & & A & $\mathrm{T}$ \\
\hline \multicolumn{5}{|l|}{ FAMILY TETRAODONTIDAE } \\
\hline Arothron hispidus (Linnaeus, 1758) & & IBUNAM & A & $\mathrm{T}$ \\
\hline Arothron meleagris (Anonymous, 1798) & $\mathrm{a}, \mathrm{c}$ & YPM & A & $\mathrm{T}$ \\
\hline Canthigaster punctatissima (Günther, 1870) & $\mathrm{a}$ & $\begin{array}{l}\text { CAS, LACM, SIO, } \\
\text { YPM, } 1\end{array}$ & A & CP-PP \\
\hline Sphoeroides annulatus (Jenyns, 1842) & a & IBUNAM, SIO & $\mathrm{C}$ & SP-PCHP \\
\hline Sphoeroides lobatus (Steindachner, 1870) & $\mathrm{a}$ & SU & $\mathrm{C}$ & SP-PCHP \\
\hline \multicolumn{5}{|l|}{ FAMILY DIODONTIDAE } \\
\hline Chilomycterus reticulatus (Linnaeus, 1758) & $\mathrm{a}$ & & $\mathrm{C}$ & $\mathrm{CT}$ \\
\hline Diodon holocanthus Linnaeus, 1758 & $a, b, c$ & IBUNAM, SIO, 1 & $\mathrm{C}$ & $\mathrm{CT}$ \\
\hline Diodon hystrix Linnaeus, 1758 & $\mathrm{a}$ & AMNH & $\mathrm{C}$ & $\mathrm{CT}$ \\
\hline \multicolumn{5}{|l|}{ FAMILY URANOSCOPIDAE } \\
\hline Kathetostoma averruncus Jordan et Bollman, 1890 & & $\mathrm{SIO}$ & $\mathrm{B}$ & OP-PP \\
\hline
\end{tabular}

Field records: $\mathrm{a}=$ visual census, $\mathrm{b}=$ gill net, $\mathrm{c}=$ charalera net; Collections records: $\mathrm{AMNH}=$ American Museum of Natural History, ANSP = Academy of Natural Sciences of Philadelphia, CAS = California Academy of Sciences; CICIMAR-CI = Centro Interdisciplinario de Ciencias Marinas del Instituto Politecnico Nacional, IBUNAM = Instituto de Biologia de la Universidad Nacional Autonoma de Mexico, LACM = Natural History Museum of Los Angeles County, MCZ = Museum of Comparative Zoology Harvard University, SIO = Scripps Institution of Oceanography, SU = Stanford University, MHNUABCS-CI = Universidad Autonoma de Baja California Sur, UMMZ = Museum of Zoology University of Michigan, USNM = National Museum of Natural History, Smithsonian Institution, YPM = Peabody Museum of Yale University; Literature records: 1 = Sanchez-Ortiz et al. (1997), 2 = Gonzalez-Cabello (unpublished*), 3 = Tavera et al. (2005), 4 = Montoya-Campos (unpublished*); Habitat: $\mathrm{A}=$ reef species, $\mathrm{B}=$ soft bottom demersal, $\mathrm{C}=$ mixed bottom demersal, $\mathrm{D}=$ pelagic-demersal, $\mathrm{E}=$ neritic-pelagic, $\mathrm{F}=$ oceanic-pelagic, $\mathrm{G}=$ mesopelagic, $\mathrm{H}=$ bathybenthonic, $\mathrm{I}=$ pelagic-benthonic; Ichthyogeographic affinity: AP = Aleutian Province, OP = Oregonian Province, $\mathrm{SP}=\mathrm{San}$ Diego Province, $\mathrm{CP}=\mathrm{Cortez}$ Province, $\mathrm{MP}=$ Mexican Province, $\mathrm{PP}=$ Panamic Province, $\mathrm{CT}=$ Circumtropical, $\mathrm{T}=$ transpacific species; $\mathrm{AM}=$ Amphiamerican species, PCHP: Peruvian-Chilean Province.

\section{RESULTS}

Based on field sampling, records from national and international online ichthyologic collections, and the scientific literature, a systematic fish check-list from San Jose and San Francisquito islands and El Pardito Islet was integrated, including 298 species representing 201 genera and assigned to 86 families, 36 orders, and two fish classes (Table 1). The families best represented in terms of the species number were the Serranidae (23 species), Gobiidae
(15 species), and Carangidae (14 species). A total of 34 families were represented by only one species. On average, there were 3.5 species per family. The most numerous genera were Lutjanus and Halichoeres with eight and six species, respectively.

The highest number of records was obtained from national and international scientific collections $(212$ species or $70.8 \%$ ); field sampling yielded 160 species records (53.7\%); and bibliographic sources yielded only 
58 species records $(19.5 \%)$. Of the 160 species recorded during field sampling, 130 (43.6\%) were recorded during visual censuses, 59 (19.8\%) species were caught in the gill net while $33(11.1 \%)$ were caught in the charalera net. The most abundant species detected by visual census (Fig. 2a) were Abudefduf troschelii ${ }^{*}$ (25.4\%), Thalassoma lucasanum (13\%), and Stegastes rectifraenum (9.5\%). The fish most abundant in the gill-net catches (Fig. 2b) were Microlepidotus inornatus (16\%), Mulloidichthys dentatus (11.4\%), and Caranx caballus (9.33\%), while for the charalera net (Fig. 2c) the dominant species were Harengula thrissina (64.4\%) and Eucinostomus dowii (11.13\%).

Fishes associated with rocky and coral reef systems were the best represented, by 124 species. Species associated with soft bottoms, mixed bottoms, and pelagic-demersal were similarly represented, by 46, 42, and 41 species, respectively (Fig. 3). In general, the fish community was dominated by a wide distribution of eurythermal tropical species of the Eastern Tropical Pacific region (22.6\%: 68 spp.; Cortez Province Panamic Province), and by species whose distribution range reaches the San Diego Province (22.2\%: 66 spp.) surpassing the northern limit of the ETP region (Fig. 4). Of the total recorded taxonomical composition, 24 species $(8.1 \%)$ were endemic to the Cortez Province. The circumtropical component was represented by 24 species $(8.1 \%)$ and the Transpacific species group was made up of 21 species $(11.1 \%)$.

\section{DISCUSSION}

The fish composition of San Jose and San Francisquito islands, and El Pardito Islet highlights the importance of these ecosystems as habitat for fish. A total of 298 species were recorded, which represent $48.2 \%$ of the total ichthyofauna (618 species) recorded for the Islands of the Gulf of California (Del Moral-Flores, unpublished $^{* *}$ ), $32.7 \%$ of the total ichthyofauna (911 species) recorded for all habitats in the Gulf of California (Hastings et al. 2010), and 23.2\% of all coastal species (1285 species) known for the Eastern Tropical Pacific (Robertson and Allen 2008).

The species richness of these islands is equivalent to that of the Islas Marías archipelago in the southeastern Gulf of California, for which 318 species are known (Erisman et al. 2011), and slightly above the 228 species known for the Islas Revillagigedos (Castro-Aguirre and Balart 2002). The variety of methods employed in the evaluation of the island's ichthyofauna allowed us to elaborate a highly representative systematic list. Of the dominant species found, the only ones taken by local fishermen and as fresh bait for fisheries are the Mexican goatfish Mulloidichthys dentatus and the wavyline grunt, Microlepidotus inornatus (see Montoya-Campos, unpublished ${ }^{* *}$ ).

Records obtained from bibliographic sources yielded only 58 species. This last result reflects the limited amount of research being carried out on these islands, even though they are important for fish biodiversity. Records from scientific collections yielded 212 species, a little over $70 \%$ of all recorded species. Museums are an invaluable source of information, product of the efforts of numerous researchers and a great quantity of evaluations carried out in several locations, with diverse methods and during different time periods (Chávez-Ramos et al. 1994, Del Moral-Flores, unpublished ${ }^{* *}$ ). Including those records increased significantly the species richness of this systematic list, which is dominated by species associated with shallow rocky and coral reef systems (41.6\% of species).

The families with the greatest species richness in this study were Serranidae (23 spp.), Gobiidae (15 spp.), and Carangidae (14 spp.). However, those families only corresponded to $41 \%, 13 \%$, and $40 \%$, respectively, of the known species in the Eastern Tropical Pacific (Robertson

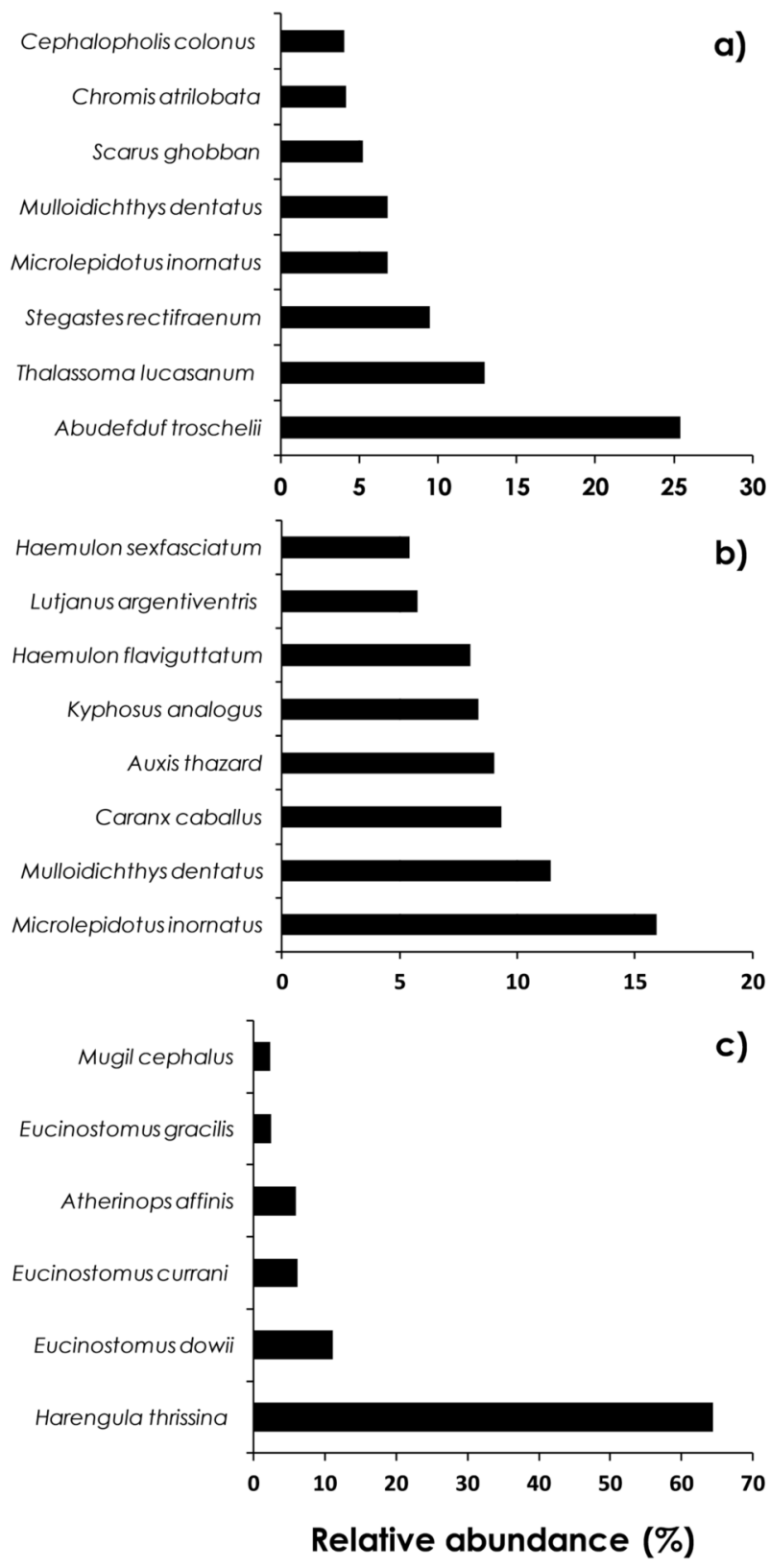

Fig. 2. Relative abundance of the most important fish species from San Jose and San Francisquito Islands, and El Pardito Islet in the southwestern Gulf of California, originally obtained with (a) visual censuses, (b) gill net captures, and (c) charalera net

\footnotetext{
${ }^{*}$ Full species names featuring the authority and the year are privided in Table 1

${ }^{* *}$ See footnote on page 178 .
} 
and Allen 2008). The more diverse family in the Eastern Tropical Pacific, such as Gobiidae with 124 species, was poorly represented in this study, this could be due to several species being endemic to the Panamic Province and oceanic islands, and several being associated to soft bottoms, which is a poorly studied habitat on the islands. Other well represented families in species richness for the region but with few species on the islands were Sciaenidae (82 spp. known in the ETP) and Ariidae (28 spp.), which prefer estuarine and lagoon systems, Cynoglossidae (18 spp.), Triglidae (10 spp.), and Bothidae ( 9 spp.) which are associated with soft bottoms on the continental platform and are abundant there, as well as Engraulidae (26 spp.) which regularly inhabit estuaries, coastal lagoons and the coastal pelagic zone.

Biogeographically, the Gulf of California is almost entirely within the Cortez Province (Hastings 2000, Robertson and Cramer 2009). Of all recorded species, 24 were endemic to this Province. Most were small seden-

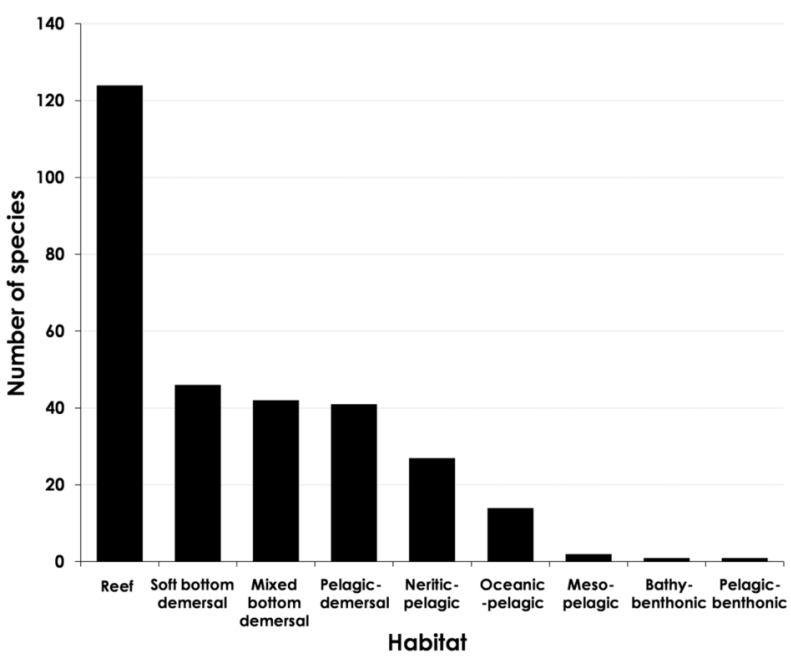

Fig. 3. Preferential habitat of fish species from San Jose and San Francisquito Islands, and El Pardito Islet in the southwestern Gulf of California

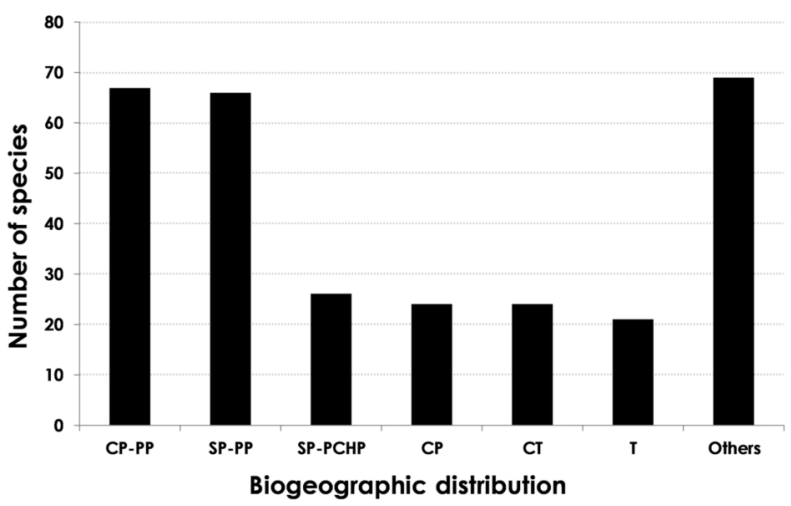

Fig. 4. Ichthyogeographic affinity of recorded species from San Jose and San Francisquito Islands, and El Pardito Islet in the southwestern Gulf of California: $\mathrm{SP}=$ San Diego Province; PP = Panamic Province; $\mathrm{PCHP}=$ Peruvian-Chilean Province; $\mathrm{CP}=$ Cortez Province; $\mathrm{CT}=$ Circumtropical; $\mathrm{T}=$ Transpacific; others $=$ all other possible combinations tary species, associated to reef systems belonging to the families Chaenopsidae (Acanthemblemaria crockeri, A. hastingsi, Coralliozetus micropes, C. rosenblatti, Emblemaria hypacanthus, E. walker, and Stathmonotus sinuscalifornici), Gobiidae (Aruma histrio, Barbulifer pantherinus, Chriolepis zebra, Elacatinus limbaughi, Gobiosoma chiquita, and Pycnomma semisquamatum), Labrisomidae (Malacoctenus hubbsi, Starksia cremnobates, and Xenomedea rhodopyga), Tripterygiidae (Axoclinus nigricaudus and Crocodilichthys gracilis), Gobiesocidae (Tomicodon boehlkei and T. humeralis), and Dactyloscopidae (Dactyloscopus pectoralis). Resident mobile species were also recorded, belonging to the families Kyphosidae (Girella simplicidens), and Opistognathidae (Opistognathus fossoris), as well as one demersal soft bottom species from the family Ophidiidae (Ophidion iris).

In general, the fish community was dominated by eurythermal tropical species widely distributed in the Eastern Tropical Pacific region (Robertson and Allen 2008). The circumtropical component was well represented by 24 species from varied habitats, including oceanicpelagic species (e.g., Carcharhinus falciformis), pelagicneritic species (e.g., Selar crumenophthalmu), demersalpelagic species (e.g., Mugil curema), mixed bottom demersal species (e.g., Diodon holocanthus), and even reef species (e.g., Fistularia commersonii). Transpacific species were only represented by reef species (e.g., Oxycirrhites typus) and pelagic-neritic species (e.g., Scomber japonicus). The majority of species from this group are reef inhabitants and have greater representation along the oceanic islands of the region (Robertson and Allen 1996, Castro-Aguirre and Balart 2002), as well as in the southern Gulf of California and the coast between Costa Rica and Panama (Thomson et al. 2000, Robertson and Allen 2008).

The evaluation of the ichthyofaunal community of these islands using several methods as well as records from bibliographic sources and scientific collections allowed us to create a systematic list with a high species richness; however, this list is still far from being complete, since some environments such as deep reefs, especially on the eastern side of the islands, have been scarcely explored or not at all. Finally, the presence of 20 elasmobranch species (10 sharks and 10 rays) near the islands and of several large species from the families Serranidae (e.g., Mycteroperca rosacea), Scaridae (e.g., Scarus perrico), and Lutjanidae (e.g., Hoplopagrus guentherii) mainly at El Pardito Islet, seems to indicate that the protective measures being carried by the local fisherman and their families that permanently inhabit this islet have contributed positively to fish conservation.

\section{ACKNOWLEDGEMENTS}

The authors thank the invaluable hospitality of the Cuevas family and fishermen from El Pardito. We wish to thank the Instituto Politécnico Nacional (COFAA, EDI) and the Centro de Investigaciones Biológicas del 
Noroeste for their support. We also thank O. Trujillo, curator from MHNUABCS-CI, for providing us with island records. DSPS and XGMS wish to thank the CONACyT program 'Complementary Support for the Institutional Strengthening Working Groups: "Retention" and for funding the projects "Análisis estructural y funcional de la ictiofauna asociada a la pesquería de camarón en el Pacífico mexicano" and "Efecto antropogenico en el cambio en la biodiversidad y funcionamiento de la ictiofauna de arrecifes rocosos de la Bahia de La Paz BCS, México".

\section{REFERENCES}

Abitia-Cárdenas L., Rodríguez-Romero F., Galván-Magaña J., De la Cruz-Agüero J., Chávez-Ramos H. 1994. Lista sistemática de la ictiofauna de Bahía de La Paz, Baja California Sur, México. Ciencias Marinas 20 (2): 159-181.

Anonymous 1978. Decreto por el que se establece una zona de reserva y refugio de aves migratorias y de la fauna silvestre en las islas que se relacionan, situadas en el Golfo de California. Diario Oficial de la Federación.

Arriaga L., Vazquez E., González J., Jiménez R., Muñoz E., Aguilar V. 1998. Regiones prioritarias marinas de Mexico. Comision Nacional para el conocimiento y uso de la Biodiversidad, Mexico.

Briggs J.C. 1974. Marine zoogeography. McGraw-Hill, New York, NY, USA.

Castro-Aguirre J.L., Balart E.F., Arvizu-Martínez J. 1995. Contribución al conocimiento del origen y distribución de la ictiofauna del Golfo de California, México. Hidrobiológica 5 (1-2): 57-78.

Castro-Aguirre J.L., Balart E.F. 2002. La ictiofauna de las Islas Revillagigedo y sus relaciones zoogeograficas, con comentarios acerca de su origen y evolucion. Pp. 153-170. In: Lozano-Vilano M.L. (ed.) Libro jubilar en honor al Dr. Salvador Contreras Balderas. Universidad Autonoma de Nuevo Leon, México.

Chávez-Ramos H., Galván-Magaña F., Abitia-Cárdenas A., De la Cruz-Agüero J., Rodríguez-Romero J. 1994. La ictiofauna marina de Baja California Sur, México, desde la perspectiva de un trabajo museológico. Investigaciones Marinas CICIMAR 9 (1): 43-49.

Chirichigno F.N., Cornejo R.M. 2001. Catálogo comentado de los peces marinos del Perú. Instituto del Mar del Perú, IMARPE, Publicación especial. Callao, Perú.

Erisman B.E., Galland G.R., Mascareñas I., Moxley J., Walker H.J., Aburto-Oropeza O., Hastings P.A., Ezcurra E. 2011. List of coastal fishes of Islas Marías archipelago, Mexico, with comments on taxonomic composition, biogeography, and abundance. Zootaxa 2011 (2985): 26-40.

Fischer W., Krupp F., Schneider W., Sommer C., Carpenter K.E., Niem V.H. 1995. Guia FAO para la identificacion de especies para los fines de pesca. Vols. 2 and 3. Pacifico Centro-Oriental. FAO. Roma. Pages: 648-1652.

Hastings P.A. 2000. Biogeography of the Tropical Eastern Pacific: Distribution and phylogeny of chaenopsid fishes. Zoological Journal of the Linnean Society 128 (3): 319-335. DOI: $10.1006 /$ zjls. 1998.0196
Hastings P.A., Findley L.T., Van der Heiden A.M. 2010. Fishes of the Gulf of California. Pp: 96-118. In: Brusca R. (ed.) The Gulf of California. Biodiversity and conservation. University Arizona Press, Tucson, AZ, USA.

Holguin-Quiñones O., Gonzalez-Medina E., Solis-Marin F., Felix-Pico E. 2008. Variacion espacio-temporal de Scleractinia, Gorgonacea, Gastropoda, Bivalvia, Cephalopoda, Asteroidea, Echinoidea y Holothuroidea de fondos someros de Isla San Jose, Golfo de California. Revista de Biologia Tropical 56 (3): 1189-1199.

Horn M.H., Allen L.G., Lea R.N. 2006. Biogeography. Pp. 3-25. In: Allen L.G., Pondella II D.J., Horn M.H. (eds.) The ecology of marine fishes: California and adjacent waters. University of California Press, Berkeley, CA, USA.

Lozano E., Carmona R., Bravata G. 2004. Exito reproductivo de la gaviota de patas amarillas (Larus livens) y de la gaviota parda (L. heermanni) en el sur del Golfo de California, Mexico. Ornitologia Neotropical 15 (2): 237-246.

Nelson J.S. 2006. Fishes of the world (4th edition). John Wiley and Sons, Hoboken, NJ, USA.

Nelson J.S., Crossman E.J., Espinosa-Perez H., Findley L.T., Gilbert C.R., Lea R.N., Williams J.D. 2004. Common and scientific names of fishes from the United States, Canada, and Mexico (6th edition). Special Publication No. 29. American Fisheries Society, Bethesda MD, USA.

Ramirez-Garcia P., Lot A. 1994. La distribucion del manglar y de los "pastos marinos" en el Golfo de California, Mexico. Anales del Instituto de Biología: Serie Botanica (UNAM) 65 (1): 63-72.

Ramirez-Rodriguez M. 1997. Produccion pesquera en la Bahia de La Paz. Pp. 273-282. In: Urban-Ramirez J., RamirezRamirez M. (eds.) La Bahia de La Paz, investigacion y conservacion. UABCS-CICIMAR-SCRIPPS, La Paz, B.C.S., Mexico.

Robertson D.R., Allen G.R. 1996. Zoogeography of the shorefish fauna of Clipperton Atoll. Coral Reefs 15 (2): 121-131. DOI: $10.1007 / \mathrm{s} 003380050032$

Robertson D.R., Allen G.R. 2008. Peces costeros del Pacífico oriental tropical online Informacion system. Version 1.0 (2008). Smithsonian Tropical Research Institute, Balboa, Panama. www.neotropicalfishes.org/sftep

Roberston D.R., Cramer K.L. 2009. Shore fishes and biogeographic subdivisions of the Tropical Eastern Pacific. Marine Ecology Progress Series 380: 1-17.

DOI: $10.3354 /$ meps07925

Robertson D.R., Grove J.S., McCosker J.E. 2004. Tropical transpacific shore fishes. Pacific Science 58 (4): 507-565. DOI: $10.1353 /$ psc.2004.0041

Rodríguez-Romero J., Abitia-Cardenas L.A., De la CruzAgüero J., Gálvan-Magaña F. 1992. Lista sistemática de los peces marinos de Bahía Concepción, Baja California Sur, México. Ciencias Marinas 18 (4): 85-95.

Sanchez-Ortiz C., Arreola-Robles J.L., Aburto-Oropeza O., Cortes-Hernández M. 1997. Peces de arrecife en la region de La Paz, BCS. Pp. 177-188. In: Urban-Ramirez J., Ramirez-Ramirez M. (eds.) La Bahia de La Paz, investigacion y conservacion. UABCS-CICIMAR-SCRIPPS, La Paz, B.C.S., Mexico. 
Tavera J.J., González-Acosta A.F., De la Cruz-Agüero J. Wiley E.O., Johnson D. 2010. A teleost classification based on 2005. First record of Seriola peruana (Actinopterygii: Carangidae) in the Gulf of California. Marine Biodiversity Records 1: e4 [No page numbers.]

Thomson D.A., Findley L.T., Kerstitch A.N. 2000. Reef fishes of the Sea of Cortez. Revised Edn. University of Texas Press, Austin, TX, USA.

Thomson D.A., Gilligan M. 2002. Rocky-shore fishes. Pp. 154-180. In: Case T.J., Cody M.L., Ezcurra E. (eds.) A new island biogeography in the Sea of Cortes. Oxford University Press, Oxford, UK. monophyletic groups. Pp. 123-182. In: Nelson J.S., Schultze H.P., Wilson M.V.H. (eds.) Origin and phylogenetic interrelationships of Teleosts.: Verlag Dr. Friedrich Pfeil, München, Germany.

Received: 3 May 2012

Accepted: 21 July 2012

Published electronically: 30 September 2012 